\title{
Risk Factors for Severe Respiratory Syncytial Virus Lower Respiratory Tract Infection
}

\author{
Constanze Sommer ${ }^{1}$, Bernhard Resch ${ }^{1, *}$ and Eric A.F. Simões ${ }^{2,3}$ \\ ${ }^{1}$ Medical University Graz, Austria \\ ${ }^{2}$ University of Colorado, Denver, and Children's Hospital Colorado, USA \\ ${ }^{3}$ University of Padjadjaran, Bandung, Indonesia
}

\begin{abstract}
RSV infection is a leading cause of lower respiratory tract infection, especially in High-risk infants with a history of prematurity, bronchopulmonary dysplasia (BPD), congenital heart disease (CHD), neuromusculair impairment, immunodeficiency, and Down syndrome. Host related risk factors that have been identified to be associated with severe RSV related lower respiratory tract infection include young age below 6 months at the beginning of RSV season, multiple birth, male sex, low socioeconomic status and parental education, crowded living conditions, young siblings, maternal smoking and indoor smoke pollution, malnutrition/small for gestational age, family history of atopy or asthma, low cord serum RSV antibody titers, and living at altitude.

Risk factors increasing the risk of acquisition of RSV have been identified to be birth before and/or during RSV season, day care attendance, presence of older siblings in school or day-care, and lack of breast feeding. Some of these risk factors are discussed controversially and some of them are found continuously throughout the literature.

Given the high cost of RSV prophylaxis, especially for the large population of late preterm infants, algorithms and risk score systems have been published that could identify high-risk infants for treatment with palivizumab out of this gestational age group. Several models reported on an average sensitivity and specificity of 70 percent and, thus, are helpful to identify infants at high risk for severe RSV infection and need for prophylaxis with palivizumab.
\end{abstract}

Keywords: Syncytial virus, immune complex reaction, IgA antibodies, RSV disease, cellular immunity.

\section{INTRODUCTION}

Respiratory syncytial virus (RSV) is a leading cause of viral lower respiratory tract infections (LRTI) in early childhood, causing an estimated 33 Million LRTI in children under the age of five, 3.4 million hospitalizations and between 66,000-199,000 deaths annually, globally [1]. Almost all infants are infected by 2 to 3 years or following two RSV seasons [2]. Infection rates peak in infants aged up to 3 months. Several hypotheses exist to explain the severity of RSV - LRTI during the first few months of life. Immunologic processes play an important role, including an immune complex reaction Type III occurring in the lung between RSV antigen and maternal acquired $\mathrm{IgG}$ antibodies [3] accompanied by a lack of secretory $\operatorname{IgA}$ antibodies, a cell mediated Type IV reaction in the lung [4] and T-cell independent mechanisms implicating macrophages and MIP $1 \alpha$ and other chemokines. Additionally the immature immune system and the small infant's airways are important factors explaining the severity of RSV disease within the first months of life [5]. This review aims to summarize the evidence for risk factors associated with severe RSV - LRTI.

*Address correspondence to this author at the Division of Neonatology, Pediatric Department Medical University hospital Graz Auenbruggerplatz 30, A- 8036 Graz, Austria; Tel: 0043316385 81134;

Fax: 0043316385 2678; E-mail: bernhard.resch@medunigraz.at

\section{PATIENT GROUPS AT HIGH RISK FOR SEVERE RSV DISEASE}

\section{Prematurity}

Prematurity goes along with small, immature and vulnerable airways, an immature immune system, an incomplete transfer of maternal antibodies and an inadequate cellular immunity which is necessary for the viral clearance. The lung development at 30 weeks gestational age (GA) reveals lung volumes being about three times smaller and a surface area being about four times smaller compared to term infants, whereas air space walls have an increased wall thickness by one third of that of term infants [6].

A longitudinal study up to 10 months of chronological age by Ballow, et al. [7]. demonstrated that hypogammaglobinemia correlated with lower gestational age and remained up to 6 months of chronological age in preterm infants below 1500 grams. Following discharge from the NICU significantly higher incidence of infections, especially LRTIs, were observed in preterm infants. Consecutively preterm infants were more often rehospitalized compared to term infants. However, despite severe hypogammaglobinemia none of the preterm infants had bacterial infection. Another study by De Sierra, et al. [8] revealed that RSV- specific antibody titers were lower in premature infants of $\leq 28$ weeks GA compared with term infants; 
and preterm infants of $\geq 29$ weeks GA had RSV- specific titers against $F$ and $G$ protein comparable to those of term infants.

Prematurity also alters the course of RSV infection. Prematurely born infants presented more often with apnoea, had a higher incidence of atelectasis/infiltrate and hyperinflation on radiographic examination, longer hospital stays, a higher physiologic stability index and therapeutic intervention score and were more likely to receive supplemental oxygen, ICU admission, mechanical ventilation and parenteral fluid therapy [9]. These findings were confirmed by a prospective study including infants younger than 24 months and being hospitalized for RSV infection [10] that revealed remarkable differences regarding length of hospital stay and severity of clinical course of disease demonstrated by higher LRI scores, more days of oxygen requirement and respiratory support between preterm and term born infants.

Horn, et al. [11] found significant differences regarding ICU admission and rate of intubation as well as length of hospital stay between preterm born and term born infants with the highest resource use in the late preterm infants of 33 - 35 weeks GA. In this study the rehospitalization rate due to RSV virus infection was $3.6 \%$.

Stevens, et al. [12] found a RSV rehospitalization rate of $7.6 \%$ in prematures born <32 weeks' gestation with the highest rate of $13,9 \%$ in the subgroup $\leq 26$ weeks gestational age. The IMPACT trial [13] confirmed these findings and revealed in infants < 35 weeks' gestation a rehospitalization rate of $8.1 \%$ in the placebo arm, in those born between 32-35 weeks' gestation this number was even higher $(9.8 \%)$. Carbonell-Estrany, et al. [14] found a rehospitalization rate of $9 \%$ in infants born $\leq 32$ weeks of gestation. However there were several studies with lower rates like studies by Resch, et al. [15] that observed a RSV rehospitalization rate of $2.5 \%$ in premature infants of 29 to 36 weeks gestation', Law, et al. [16] with a rate of 3.6\% in infants of 33-35 weeks' gestation and Figueras-Aloy, et al. [17] with a rate of $3.7 \%$ in infants of 33-35 weeks' gestation.

Recent data by Gunville, et al. [18] showed again a higher rate of $6.6 \%(18 / 271)$ of children aged under 2 years born prematurely (<36 weeks' gestation) and being rehospitalized due to RSV disease. In the subgroup analysis $2.6 \%$ were below 32 weeks' gestation and $4.1 \%$ were of 32 35 weeks' gestation.

A summary of the data is shown in Table $\mathbf{1 .}$

\section{Bronchopulmonary Dysplasia (BPD)}

Ventilated preterm infants, especially those below $1000 \mathrm{~g}$ birth weight, are at high risk for developing BPD. In these infants ventilation-induced volutrauma and oxygen toxicity as well as pre- and postnatal infections can initiate and sustain a pulmonary inflammatory response leading to a possible impaired development of the lung [19] Groothuis, et al. [20] were the first to study the burden of early rehospitalization in a prospective study including 30 infants

Table 1. Prematurity and RSV-Related Rehospitalization Rates

\begin{tabular}{|c|c|c|c|c|c|}
\hline $1995-1996$ & 11 & USA & $\begin{array}{l}<37 \mathrm{wk} \\
\leq 32 \mathrm{wk} \\
33-35 \mathrm{wk} \\
36 \mathrm{wk}\end{array}$ & 304 & $\begin{array}{l}3,6 \%(11) \\
1,3 \%(4) \\
1,6 \%(5) \\
0,7 \%(2)\end{array}$ \\
\hline $1996-1997$ & 13 & USA & $\begin{array}{l}<35 \mathrm{wk} \\
32-25 \mathrm{wk} \\
<32 \mathrm{wk}\end{array}$ & 1502 & $\begin{array}{l}8,1 \% \\
9,8 \% \\
11 \%\end{array}$ \\
\hline 1998-1999 & 14 & Spain & $\leq 32 \mathrm{wk}$ & 584 & $9 \%(53)$ \\
\hline $1999-2000$ & 15 & Austria & $<37$ wk & 281 & $2,5 \%(7)$ \\
\hline $2000-2002$ & 16 & Canada & $33-35 \mathrm{wk}$ & 1832 & $3,6 \%(66)$ \\
\hline $2005-2007$ & 17 & Spain & $33-35 \mathrm{wk}$ & 5441 & $3,7 \%(202)$ \\
\hline $2006-2007$ & 18 & USA & $\begin{array}{l}<36 \mathrm{wk} \\
<32 \mathrm{wk} \\
33-35 \mathrm{wk}\end{array}$ & 271 & $\begin{array}{l}6,6 \%(18) \\
2,6 \%(7) \\
4,1 \%(11)\end{array}$ \\
\hline
\end{tabular}


$<2$ years with BPD receiving home oxygen therapy after discharge from the hospital. In this group 11 of 15 hospitalizations were due to an RSV infection, $36 \%$ of the infants were admitted to the intensive care unit (ICU) $18 \%$ of whom required mechanical ventilation. In the PREVENT Study [21], a randomized placebo controlled trial on the use of intravenous immunoglobulin prophylaxis (RSV- IGIV) in preterm infants with and without BPD, $17.4 \%$ infants with BPD (26/149) were rehospitalized due to proven RSV infection. The IMpact- study [22], a randomized, double- blind, placebo controlled trial conducted in the United States, Canada and the United Kingdom determining the safety and efficiency of palivizumab, revealed an RSV- rehospitalisation rate of $12.8 \%$ in infants with BPD, which was reduced to $7.9 \%$ (39\% reduction) in the group having received palivizumab prophylaxis. Thereafter several other studies revealed RSV hospitalization rates ranging up to $24.4 \%$ [11, 14, 23, 24]. Stevens, et al. [11] reported in a cohort of 131 infants requiring respiratory support beyond 36 weeks postconceptional age that $24.4 \%$ were rehospitalized due to RSV infection. They were also more likely to be admitted with RSV than infants with respiratory support up to 36 weeks postconceptional age. In the study of Carbonell- Estrany [14] RSV rehospitalization rate was $15 \%$ in infants with chronic lung disease. In the multivariate logistic regression model the higher risk for hospital admission in infants with chronic lung disease was a significant prognostic variable $(\mathrm{p}<0,016)$.

Greenough, et al. [24] reported in retrospective review that in a study cohort of 235 neaontes prematurely born neonates < 32 weeks' gestation requiring oxygen beyong 28 days after birth $19 \%$ were readmitted to the hospital due to a proven RSV infection.

A more recent study from The Munich RSV Study Group [25] reported on a rehospitalisation rate of $15.4 \%$ in preterm infants $\leq 35 \mathrm{GA}$ with $\mathrm{BPD}$. In the multivariate logistic regression model BPD as well as male sex and day careattendance of siblings yielded statistical significance as an independent predictor for RSV rehospitalisation.

Another prospective German multicentre study from the RSV Paed Study Group [26] found RSV hospitalization rates of $12.3 \%(50 / 356)$ in preterm infants with BPD. The authors also reported on a significantly higher proportion of nosocomially acquired RSV infections in the BPD group $(30.8 \%$ vs. $9.8 \%, \mathrm{p}<0.001)$. In the univariant analysis the criterion premature infant having BPD requiring treatment over the last months was significantly associated with a complicated clinical course and was significantly and independently associated with the combined outcome "complicated course of disease" in the multivariate analysis. A summary is shown in Table 2 .

\section{Congenital Heart Disease (CHD)}

Infants with CHD are known to be at increased risk of severe illness from RSV infection regarding higher morbidity (more complicated clinical course, e.g. need for assisted ventilation or longer duration of oxygen supplementation) and higher mortality rate that first was reported by MacDonald, et al. [27] to be as high as 37\%. Navas, et al. [28] from the PICNIC Study Group in Canada reported on $260(16.4 \%)$ out of 1584 patients with RSV infection having underlying cardiac disease. These children required oxygen supplement for a significant longer time period and had a significantly higher mortalitiy rate $(3.4 \%)$ compared to children without cardiac disease. In a following study from the PICNIC Study Group Wang, et al. [29] reported on a lower rate of $8.2 \%(57 / 689)$ of children with underlying cardiac disease showing a mortality rate of $5.3 \%$.. In the Cardiac Trial (1998) [30] the use of RSV hyperimmune globulin in the prevention of severe RSV infection was associated with increased mortality, thus, the newly developed humanized monoclonal antibody, palivizumab, had to be proven to be safe in this particular population. Feltes, et al. (2003) [31] published data of a multicenter, randomized, double-blind, placebo-controlled trial of 1287 children with hemodynamically significant CHD reporting on an RSV hospitalization rate of 9.7\% (63/648) that was impressively reduced by $45 \%$ using monthly palivizumab prophylaxis. Based on this data an AAP policy statement was released in 2003 [32] recommending RSV prophylaxis with palivizumab for children $<24$ months with underlying hemodynamically significant CHD. This recommendation was also outlined in the revised AAP guidelines in 2009 [33]. The Palivizumab Outcome Registry Group (2008) [34] collected data on 1500 children with $\mathrm{CDH}$ accounting for $7.7 \%$ of the entire registry cohort of 19548 , who received

Table 2. Chronic Lung Disease (CLD)/ Bronchopulmonary Dysplasia (BPD) and RSV- Related Rehospitalization Rates

\begin{tabular}{|c|c|c|c|c|c|}
\hline 1985-1986 & 20 & USA & $59 \%(16)$ & 30 & $0 \%$ \\
\hline 1996 & 22 & USA, Canada, UK & $12,8 \%(34)$ & 266 & $1,0 \%(5)$ \\
\hline 1994-1997 & 24 & UK & $19 \%(45)$ & 235 & - \\
\hline 1998- 1999 & 25 & Germany & $15,4 \%(8)$ & 53 & - \\
\hline 1999-2005 & 26 & Germany & $12,3 \%(50)$ & 356 & $8,0 \%(4)$ \\
\hline
\end{tabular}


RSV prophylaxis with palivizumab over four seasons (20002004). The majority (71\%) had acyanotic CDH. Overall, only $1,9 \%$ exhibited rehospitalization due to laboratoryconfirmed RSV infection. The hospitalization rate was significantly higher for subjects with compared to those without diagnosis of $\mathrm{CDH}(1.9 \%$ vs. $1.2 \%, \mathrm{p}<0,03)$, but was significantly lower compared to former trials. A recent California state-wide study by Chang, et al. (2010) [35] on the use of palivizumab in $\mathrm{CDH}$ demonstrated a $19 \%$ reduction rate of RSV related hospitalizations being equal to seven fewer RSV hospitalizations per year for patients with hemodynamically significant CHD. In a Danish study [36] predictors of RSV hospitalization included Down syndrome (OR 3.24, 95\% CI 1.80 to 5.80), cardiomyopathy (odds ratio (OR) $5.84,95 \%$ CI 1.26 to 27.16 ) and hemodynamically significant heart disease (OR $1.53,95 \%$ CI 1.04 to 2.26 ) by multivariate analysis. Predictors for the need of respiratory support during RSV hospitalization including supplemental oxygen, nasal continuous positive airway pressure or mechanical ventilation were young age (relative risk (RR) $0.47,95 \%$ CI 0.32 to 0.67 per additional year of age) and cardiac decompensation (RR 1.81, 95\% CI 1.02 to 3.23).

Table 3 summarizes rehospitalization rates due to RSV in children with CHD.

\section{Neuromuscular Impairment}

Another group of infants being at increased risk for severe RSV infection are those with neuromuscular impairment. Factors predisposing to a more severe course of RSV disease in neuromuscular disease include the impaired ability to clear secretions from the airways due to ineffective cough resulting from respiratory muscle weakness and the high prevalence of gastro-oesophageal reflux and swallowing dysfunction, which leads to aspiration [37]. Several studies have proven the increased morbidity and mortality in patients with an underlying neurological impairment. Based on these data recommendations for RSV prophylaxis in this high risk group have been integrated in the AAP guidelines published 2009 [33]. A more detailed essay is the subject of another chapter in this supplement.

\section{Immunodeficiency, Immunosuppression}

Patients with severe combined immune deficiency syndrome and those with acquired immune deficiency syndrome appear to be highly susceptible to severe, persistent infections due to a variety of microorganisms, of which viruses likely are the most common. Infants show prolonged viral shedding and increased morbidity and mortality rates associated with RSV infection [37]. RSV was recognized as a cause of severe disease following bone marrow transplantation (BMT). The presence of neutropenia and lymphopenia was identified as being risk factors for the development of LRTI infections. A phase-1 study among adult recipients of hematopoietic stem cell transplantation (HSCT) showed that intravenous palivizumab was well tolerated among HSCT patients with and without RSV infection [38]. In infants a decision analysis model of palivizumab prophylaxis to prevent RSV related mortality after pediatric BMT revealed a $10 \%$ increase in survival in infants who had received palivizumab. The absolute survival rate increased from $83 \%$ to $92 \%$ [39]. Hall, et al. (1986) [40] described a cohort of immunocompromized children receiving anticancer therapy and found prolonged viral shedding as well as higher incidences of LRTI and a higher mortality rate (15\%) compared to immune competent children. Cole et al (2001) [41] also reported on a common finding of prolonged viral shedding in 18 children with cancer and laboratory confirmed RSV infection. Mortality related to RSV infection was low independent whether or not specific antiviral therapy was given. These findings were confirmed by King, et al. (1993) [42] in infants with human acquired immunodeficiency syndrome (HIV) and RSV infection. However, a more recent study from South Africa [43] found HIV infected children being at increased risk of viral LRTI presenting more often as viral pneumonia and having a higher mortality rate compared to not HIV infected children. Additionally, HIV- infected children showed a less seasonal associated occurrence of RSV infection than non-infected infants.

Overall these data suggest that infants following solid organ or HSC transplantation, infants with congenital or acquired immunodeficiency and those under immunosuppressive therapy are a possible new target group to for RSV prophylaxis with palivizumab.

A summary is displayed in Table 4.

\section{Down Syndrome}

Down syndrome is the most common chromosomal abnormalitiy and due to it's variability of immunulogic impairments and concomitant respiratory patholoy it is associated with a high risk of respiratory tract infections.

Table 3. Congenital Heart Disease (CHD) and RSV- Related Rehospitalization Rates

\begin{tabular}{|l|l|l|l|l|l|}
\hline Study Years & Reference No. & Country & RSV + CHD (No.) & Total No. of Patients & Mortality Rate (No.) \\
\hline \hline $1976-1980$ & 27 & USA & $11,8 \%(27)$ & 229 & $37 \%(10)$ \\
\hline $1988-1991$ & 28 & Canada & $16,4 \%(260)$ & 1584 & $3,5 \%(9)$ \\
\hline 1993 & 29 & Canada & $8,3 \%(57)$ & 689 & $5,3 \%(3)$ \\
\hline $1998-2002$ & 30 & Multicenter & $9,7 \%(63)$ & 648 & $6,3 \%(4)$ \\
\hline $2000-2006$ & 36 & USA & $3,0 \%(1596)$ & 53207 & $1,9 \%(10)$ \\
\hline $1996-2003$ & 37 & Denmark & $9,7 \%(313)$ & 3239 & none \\
\hline
\end{tabular}


Table 4. Immunosuppression/ Immunodeficiency and RSV- Related Hospitalization Rates

\begin{tabular}{|l|l|l|l|l|l|}
\hline Study Years & $\begin{array}{l}\text { Reference } \\
\text { No. }\end{array}$ & Country & $\begin{array}{l}\text { Immunosuppression + } \\
\text { RSV (No.) }\end{array}$ & $\begin{array}{l}\text { Total No. of } \\
\text { Patients }\end{array}$ & Underlying Disease \\
\hline \hline $1974-1984$ & 41 & USA & $7,7 \%(47)$ & 608 & $\begin{array}{l}\text { Immune compromized (chemotherapy, cortico-steroid } \\
\text { therapy, immuno-deficiency disease) }\end{array}$ \\
\hline $1994-1998$ & 42 & USA & $(18)$ & unknown & Cancer with polychemotherapy \\
\hline $1990-1993$ & 43 & USA & $7,6 \%(10)$ & 131 & HIV \\
\hline $1997-1998$ & 44 & South Africa & $5,3 \%(23)$ & 433 & HIV \\
\hline
\end{tabular}

Bloemers, et al. (2007) [44] reported an incidence of 9,9\% in children with down syndrome. The rate of hospitalization for RSV was lower in children with Down snydrome without chronic heart disease $(7,6 \%)$ than in preterm children with Down syndrome $(9,4 \%)$ or children with Down syndrome and significant chronic heart disease (11,9\%). Disease severity was not different in children with and without additional risk factors such as prematurity or chronic heart disease. In contrast Hilton, et al. (1999) suspected that chronic heart disease does not influence the high admission rate to hospital in children with Down syndrome, but seems to increase the severity of disease. Kristensen (2009) [36] also showed in a multivariante analysis that children with Down syndrome had an increased risk of hospitalization of RSV infection. A very recent study by Bloemers, et al. (2010) [45] showed that hospitalization for RSV induced respiratory tract infections in children with Down syndrome did not significantly increase the risk of long- term airway morbidity, whereas in children without Down syndrome the reportet association between development of recurrent wheezes and severe RSV related respiratory tract infection was confirmed. Unexpectantly a high incidence of recurrant wheezes in children with Down syndrome, both with and without history of RSV related respiratory tract infection, was found.

\section{HOST RELATED RISK FACTORS INCREASING SEVERITIY OF RSV INFECTION}

\section{Age $<6$ Months at the Beginning of the RSV Season}

Young age at the beginning of the RSV season is a risk factor for both the development of LRTI as well as hospitalization due to RSV infection. Several factors play an important role including the immature immune system, narrow airways and a bias toward a TH2-type response. Law, et al. (1998) [46] showed that age below 12 weeks and a GA below 37 weeks contributed independently to the severity of RSV infection. A review of recent studies of RSV hospitalization rates [47] revealed that approximately $10 \%$ to $28 \%$ of infants hospitalized with RSV are aged below 6 weeks, $49 \%$ to $70 \%$ below 6 months, and $66 \%$ to $100 \%$ below 1 year. The greatest risk factor for hospitalization due to RSV infection appears to be the first few months of life when they coincide with the first half of the RSV season. This data was also confirmed by two Spanish studies from the IRIS Group [17, 48] showing that infants with a chronological age below 10 weeks at the onset of the RSV season were at higher risk for RSV related hospitalization.

\section{Multiple Birth}

In a study cohort from Denver between 1993 and 1989 twins and triplets were reported to have a significantly higher risk of sever RSV LRTI and higher rates of RSV related hospitalizations compared to matched singletons [49]. This finding was confirmed by a study on hospitalization rates in preterm infants aged 29 to 36 weeks by Resch, et al. in 2005 [15] that revealed multiple gestation being a risk factor for RSV related hospitalization (odds ratio 5,500, CI 95\% 1.439- 21.028). In the Spanish FLIP and FLIP- 2 studies $[17,48]$ multiple birth was not associated with higher rates of RSV related hospitalizations. However, the authors did not perform a multivariate regression analysis.

\section{Male Sex}

Male sex is known to be a risk factor for severe RSV LRTI. An analysis of representative studies over the last 30 years found the risk ratio of boys to girls being 1.425:1 [47]. The reason therefore seems to be of anatomic nature that boys have shorter and narrower airways and are more likely to develop bronchial obstruction in case of RSV infection. Whereas male sex was regarded as a strong and independent risk factor for RSV related hospitalization in the Canadian PICNIC study [16] this finding could not be confirmed by the data of the Spanish FLIP study [48].

\section{Low Socioeconomic Status and Parental Education}

Less parental education and low socioeconomic status have also been reported to be risk factors for RSV infection [50]. Only one study from Sweden in 2002 [51] examined the role of socioeconomic status in a non-individual setting in detail showing a positive correlation with the percentage of immigrants and per capita income by univariate analysis. Several studies $[48,52,53]$ however, could not confirm the association of less- educated mothers or parents with an increased risk for RSV LRTI. In combination with other risk factors maternal education was positively correlated with RSV related hospitalization in an early epidemiologic study from Tucson in 1991 [54].

\section{Crowded Living Conditions /Siblings}

Both crowded living conditions and siblings appear to be important risk factors for more severe RSV LRTI and RSV related hospitalization. Reasons therefore include the increased likelihood of exposure to the virus and subsequently the increased risk for infection. Many studies demonstrated a significant effect of increased numbers of 
persons sharing a bedroom on RSV LRTI [47]. This effect was increased in families with low maternal education and even more in families with low maternal education who had not breast-fed their babies. School- and preschool-aged siblings carry an increased for the young infant to acquire RSV infection. In the Canadian PICNIC study [16] the presence of preschool-aged siblings was significantly and independently associated with an increased risk for RSV related hospitalization, and a weaker association was found with the presence of school-aged siblings. Crowding, defined as 5 or more people living in one household, was also demonstrated to be a significant risk factor for RSV related hospitalization. In some contrast the Spanish FLIP study [48] revealed that only school-aged siblings and the presence of more than 4 additional residents and visitors at home were risk factors significantly associated with RSV related hospitalization. In the FLIP-2 study [17] the effect of schoolaged siblings was confirmed but not crowding by use of the same definition. The Munich RSV Study Group [25] found that siblings at day care attendance significantly augmented the risk for RSV related hospitalization.

\section{Maternal Smoking and Indoor Smoke Pollution}

Studies on the influence of smoking in the household on the risk of bronchiolitis and RSV LRTI associated hospitalization are conflicting $[47,55]$. The Spanish FLIP [48] and FLIP-2 [17] studies revealed that the presence of more than two smokers at home was associated with a higher risk of RSV related hospitalization by bivariate analysis. Also The Canadian PICNIC- Study [16] reported household exposure to cigarette smoke from 2 or more smokers being predictive for RSV related hospitalization. The inconsistent evidence on the effect of tobacco smoke exposure on RSV LRTI needs further evaluation, and hence, the AAP stated that the term air pollution better might characterize this risk factor [33].

\section{Malnutrition/Small for Gestational Age}

The role of malnutrition has not been studied in industrialized nations. The only existing studies looking at these risk factors were carried out in developing countries. All of them conclude that RSV was less frequently diagnosed in malnourished compared to well-nourished children. Thus, malnutrition has not been proven to be a risk factor for the development of RSV LRTI. The suspected reason for that phenomenon might be that a lower immune response provides protection against severe RSV disease [47]. Although low birth weight is recognized as a risk factor for severe RSV disease the role of intrauterine growth restriction has only been examined in the Canadian PICNIC- Study [16] being an independent risk factor for hospitalization for RSV infection. However, intrauterine growth restriction was found to be a risk for death due to RSV disease in developing countries [47].

\section{Family History of Atopy of Asthma}

The association of RSV infection with a family history of allergic disorders is discussed controversial. On the one hand RSV is a major cause of recurrent wheezing and "asthma" during the following years after an first severe RSV LRTI, on the other hand a disposition to asthma causing recurrent wheezing is also a cause of debate. In the Canadian PICNICstudy [16] a history of eczema in a first degree family member was found to be an independent protective factor for RSV hospitalization. However, no association was found for family history wheezing or any other allergic disorder. Data from the Spanish FLIP-2- study [17] confirmed these findings showing that a family history of wheezing did not reach statistical significance. In the previous FLIP- study [48] however, the history of wheezing in the family was found to be of statistical significance, whereas the interaction between a history of asthma or eczema in the family did not reach statistical significance in the multivariate logistic regression analysis.

\section{Low cord Serum Antibody Titers}

High titers of maternally derived RSV neutralizing antibodies are inversely associated with the incidence of acute RSV LRI during the first six months of life. In preterm infants incomplete maternally antibody transfer has been implicated to increase the risk for severe RSV infection [56]. RSV peak activity during winter epidemics appear to be associated with decreased herd immunity in the population, especially among mothers. In case of high maternal antibody titers during the latter part of the RSV season, infants born during this period might be protected. Babies conceived at the end of a RSV season may have lower antibody titers due to a natural decline of antibodies during the year and thus might be more susceptible to RSV disease at the time of birth [57] A recent study from Stensballe, et al. (2009) [56] showed a temporal association of cord- blood RSV antibody levels and rate of hospitalization for RSV in infants under 6 months of age in Denmark. The incidence of RSV hospitalization increased when RSV antibody titers (to the log base 2) declined below 7.5, and the RSV epidemic peaked shortly after the nadir of the mean antibody level

\section{Living at Altitude}

An analysis comparing hospitalization rates for children at 3 altitude categories $(<1500 \mathrm{~m} ; 1500-2500 \mathrm{~m}$; and $>2500$ $\mathrm{m})$ found that infants living at altitudes higher than $2500 \mathrm{~m}$ were $33 \%$ more likely to be hospitalized (relative risk [RR]: $1.30 ; \mathrm{P}<.018)$ than those at moderate altitudes, with $1-$ to $4-$ year-old children exhibiting an $80 \%$ increase in their hospitalization rates (RR: 1.80; P <.001) [58]. Altitude can have numerous respiratory effects including lower oxygen saturation values, impaired ciliary activity, and hypoxiarelated pulmonary vasoconstriction.

A summary of the host related risk factors of RSV is displayed in Table $\mathbf{5}$.

\section{RISK FACTORS FOR ACQUISITION OF RSV}

\section{Birth before or during RSV Season}

As mentioned above birth during the first half of the RSV season is a risk factor for RSV LRTI requiring hospitalization. One aspect is that birth shortly before and early after onset of RSV season results in a longer exposure period to RSV at a very young age. Another aspect is the fact that maternal antibodies titers to RSV show a seasonal 
Table 5. Host Related Risk Factors for RSV Infection

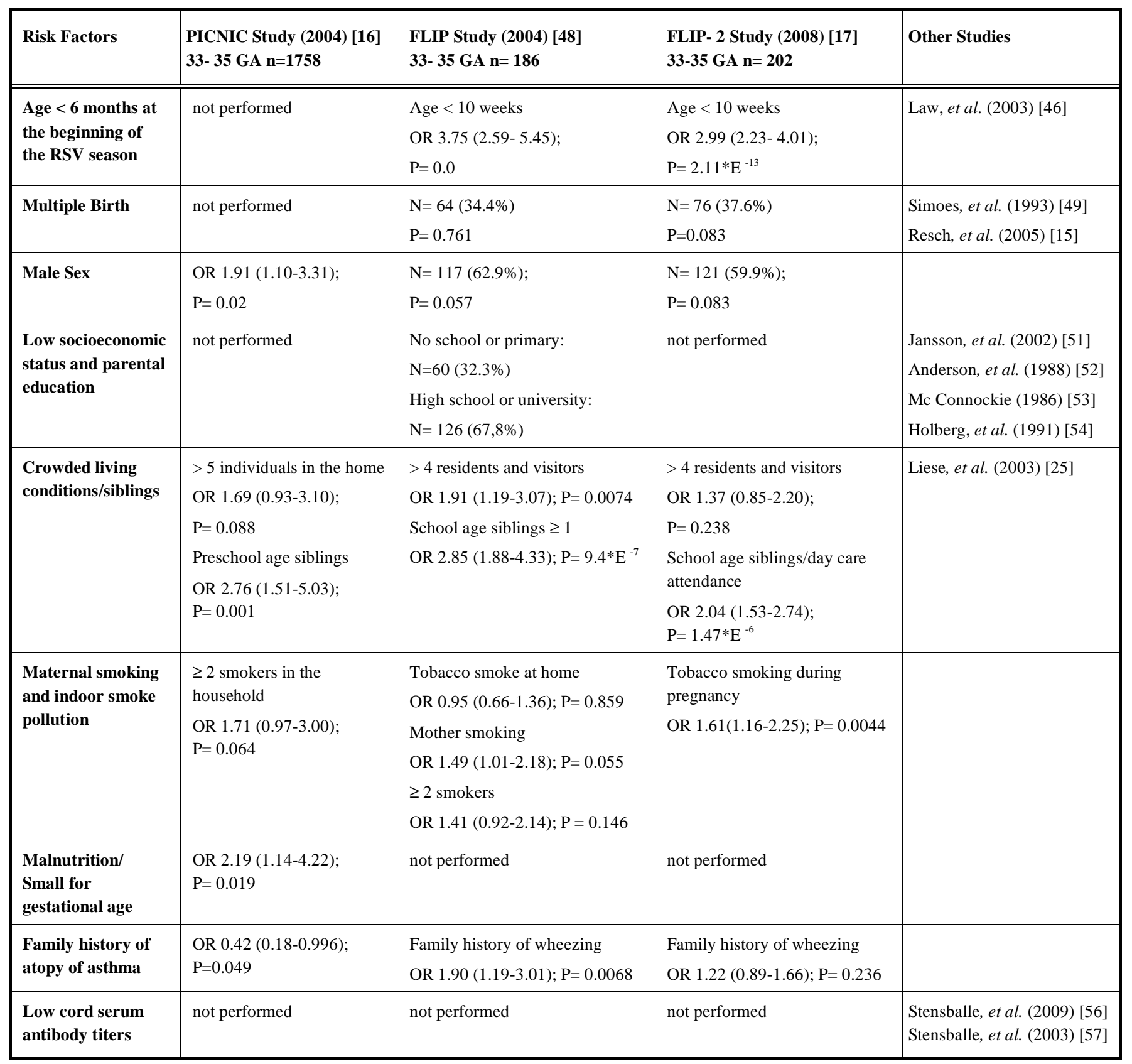

OR Odds ratio; $\mathrm{P}$ p-value, $\mathrm{N}$ number, GA gestational age.

variation with lower levels within the first half of the RSV season due to the fact that mothers have not been exposed to RSV for a longer time period. In the Canadian PICNIC study [41] the logistic regression analysis showed that birth between November and January was one of the five independent predictors for the increased risk of RSV hospitalization. This data was also confirmed by the Munich RSV Study Group [19] Risk factor analysis revealed that discharge between October and December was associated with a higher risk for RSV hospitalization (OR 2.1; 0,99- 4,4; $\mathrm{P}=0,05)$. The two Spanish FLIP and FLIP- 2 studies, [38, 39] however, demonstrated that infants with a chronological age below 3 months or less at onset of RSV season were at higher risk for RSV hospitalization.
Discharge from the NICU during RSV season was not a single significant risk factor.

\section{Day Care Attendance/ Older Siblings in School or Day-Care}

In the past the attendance of infants and young children in a child care setting or a day care group was regarded as a significant risk factor for acquisition of LRTI. Henderson, et al. (1979) [50] already described that nearly all 103 healthy children, who did not have prior exposure to RSV, became infected in a day- care setting during a 10- year time period. Several further studies confirmed this association [37] Liese, et al. [19] from the Munich RSV Study Group 
found that the presence of siblings in day-care attendance increased the risk factor for RSV rehospitalisation in preterm infants $\leq 35$ weeks gestational age significantly, whereas daycare attendance of the child itself was not a significant risk factor. In the Canadian PICNIC study [41] day-careattendance of children was the single greatest risk factor in preterm infants born at a gestational age of 33- 35 weeks, whereas the two Spanish studies- FLIP and FLIP-2 study [38,39] revealed no significant association between RSV hospitalization and day- care attendance in this age group. This was partly explained by the limited utilization of daycare centres in the study population $(<5 \%)$.

\section{Lack of Breast Feeding}

The role of breast-feeding in preventing RSV disease and hospitalization for RSV is undisputed. However data about the specific protective effect of breast- feeding on RSV infection are conflicting. The reason for its protective effect is attributed to RSV- $\operatorname{IgA}$ and lactoferrin in the breast milk as well as to the fact that breast milk promotes maturation probably through the influence of prolactin. The Spanish FLIP- Study [38] found breast feeding for $>2$ months protective against RSV hospitalization whereas the FLIP-2 Study [39] did not show a protective effect against RSV. Again in the Canadian PICNIC - Study [41] absence of breast feeding was not found to be an independent predictor for RSV-infection. However, some studies indicate that the absence of breast-feeding in combination with other risk factors like crowding, passive smoke exposure or low socioeconomic status significantly increases the risk for development of RSV LRTI [37].

A summary of risk factors for acquisition of RSV is displayed in Table 6.

\section{MODELS FOR PREDICTING RSV IN THE LATE PRETERM INFANTS}

Given the high cost of RSV prophylaxis, especially for the large population of late preterm infants, an algorithm that could identify high-risk infants with significant specificity and sensitivity could support the evidence base for recommendations for treatment of this group. Since the AAP had already made broad recommendations for prophylaxis of children in the 33-35 wGA group, we aimed to develop an European model for predicting which premature infants 33 to 35 weeks gestational age were at highest risk for hospitalization. Initially we used data from Spain and Germany [58] to develop the model then we validated the scoring system using studies from Denmark [59], France [60] and Italy[61].

The model assigns individual weights to the 7 most predictive factors from the FLIP study: birth \pm 10 weeks from the beginning of the RSV season; birth weight; breast fed 2 or fewer months; number of siblings aged 2 years or older; number of family members with atopy; number of family members with wheezing; and sex [59,60,61,62]. In the original study, information on these 7 factors examined by discriminant function analysis resulted in a diagnostic accuracy of $71 \%$ when trying to identify premature infants hospitalized with RSV. Discriminant function analysis optimizes which variables discriminate between two or more naturally occurring groups, here RSV-hospitalized vs. nonRSV-hospitalized premature infants 33 to $35 \mathrm{wGA}$. The summary diagnostic accuracies of the initial model derived from the FLIP 2 study as well as the validations from Munich, France, Italy and Denmark are presented in Table 7. It is currently being implemented in some countries to determine the most effective application of palivizumab in this population.

Sampalis, et al. [63] also developed a scoring system, based on a logistic regression model, using prospectively collected data on 1758 Canadian infants 33-35wGA, 66 of whom were hospitalized,and validated using the FLIP 2 study data. The risk score also included 7 risk factors and cutoff scores of 0-48, 49-64, and 65- 100 for low-, moderate-, and high-risk subjects, respectively. The sensitivity and specificity for predicting RSV hospitalization were $68 \%$ and $72 \%$, respectively, with the Canadian data and $62 \%$ and

Table 6. Risk Factors for RSV Acquisition

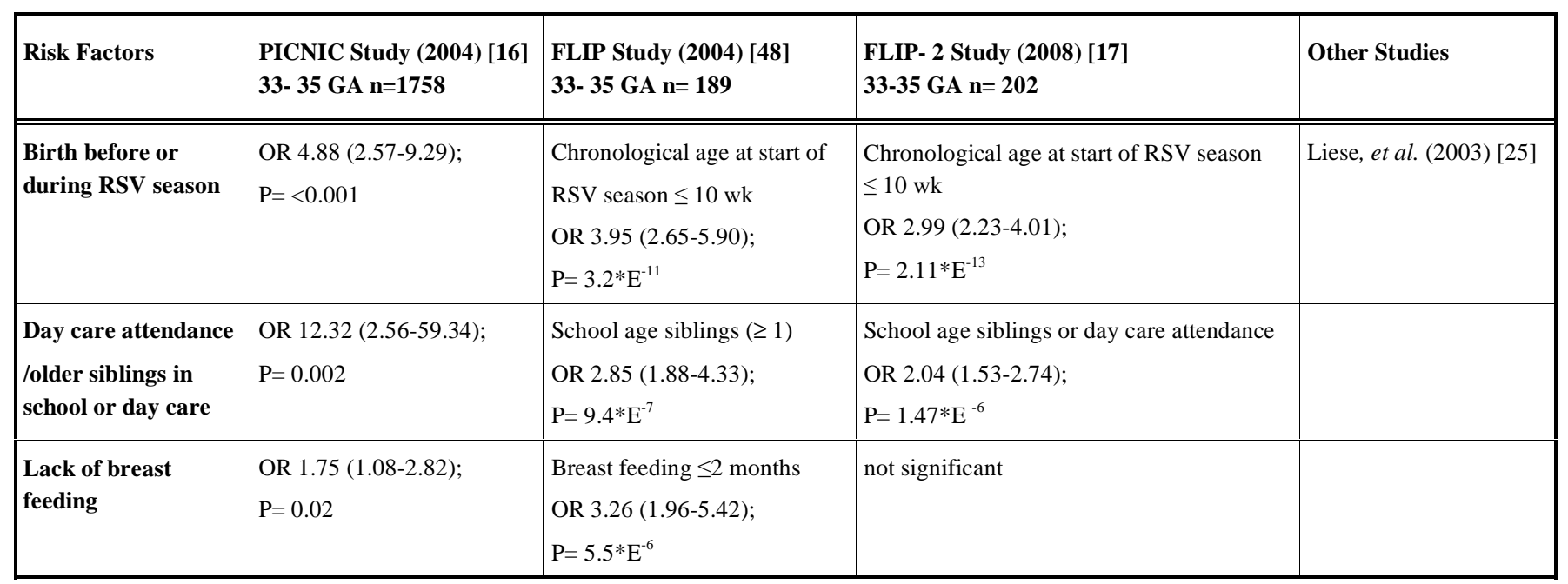

OR Odds ratio; $\mathrm{P}$ p-value, $\mathrm{N}$ number, GA gestational age. 
Table 7. European Risk Factor Scoring Models and their Diagnostic Values

\begin{tabular}{|l|l|l|l|l|l|l|l|l|}
\hline & Reference & $\mathbf{N}$ & Sensitivity & Specificity & PPV \% & NPV \% & LR & Diagnostic Accuracy \% \\
\hline \hline FLIP 15 variable modelf & 59 & 550 & 0.71 & 0.72 & 56 & 83 & 2.56 & 72 \\
\hline FLIP Final 8 variable model & 59 & 489 & 0.75 & 0.72 & 58 & 84 & 2.66 & 73 \\
\hline Munich 7 variable model & 59 & 370 & 0.88 & 0.72 & 13 & 99 & 3.17 & 73 \\
\hline French 7 variable model & 61 & 231 & 0.51 & 0.68 & 44 & 73 & 1.59 & 63 \\
\hline $\begin{array}{l}\text { French 8 variable model including } \\
\text { 'pets at home' }\end{array}$ & 61 & 231 & 0.69 & 0.63 & 48 & 80 & 1.86 & 65 \\
\hline $\begin{array}{l}\text { French 8 variable model including } \\
\text { 'day care outside the home' }\end{array}$ & 61 & 231 & 0.60 & 0.64 & 46 & 76 & 1.67 & 63 \\
\hline $\begin{array}{l}\text { French 8 variable model including } \\
\text { 'number of smokers' }\end{array}$ & 61 & 231 & 0.53 & 0.64 & 42 & 73 & 1.46 & 60 \\
\hline Italian 6 variable model & 62 & 56 & 0.83 & 0.70 & 75 & 79 & 2.79 & 77 \\
\hline Danish 7 Variable Model & 60 & 2529 & & & & & 66 \\
\hline
\end{tabular}

PPV $=$ positive predictive value

$\mathrm{NPV}=$ negative predictive value

$\mathrm{LR}=$ likelihood ratio of a positive test

Number of children at school was used in place of number of siblings over two years old.

$66 \%$, respectively, with the FLIP data set. In a single center study using this scoring tool [64], in 430 children over 3 seasons, 346 infants in the low risk group received no prophylaxis, while 78 of 84 moderate to high risk infants received prophylaxis. None of the latter group was hospitalized, but 5/346 low risk group infants were hospitalized. Thus it would appear that the Canadian scoring tool identified most high risk babies for prophylaxis since the overall rate of hospitalization was 5/430 (1.2\%) compared to the rate of 66/1758 (3.7\%) [65] in the multicenter study of un-prophylaxed Canadian infants. Validation of the European Risk Factor Scoring Tool is being undertaken in Holland and other European countries.

\section{REFERENCES}

[1] Nair H, Nokesc DJ, Gessnere BD, et al. Global burden of acute lower respiratory infections due to respiratory syncytial virus in young children: a systematic review and meta-analysis. Lancet 2010; 375: 1545-55

[2] Glezen WP, Taber LH, Frank AL, Kasel JA. Risk of primary infection and reinfection with respiratory syncytial virus. Am J Dis Child 1986; 140: 543-6.

[3] Delgado MF, Coviello S, Monsalvo AC, et al. Lack of antibody affinity maturation due to poor Toll-like receptor stimulation leads to enhanced respiratory syncytial virus disease. Nat Med 2009; 15: 34-41.

[4] Schmidt AC, Johnson TR, Openshaw PJM, et al. Respiratory syncytial virus and other pneumoviruses: a review of the international symposium-RSV 2003. Virus Res 2004; 106: 1-13.

[5] Hall CB. Respiratory syncytial virus. textbook of pediatric infectious diseases. $5^{\text {th }}$ ed. 2004; 185A: 2315-41.

[6] Wert SE. Normal and abnormal structural development of the lung. In: Polin RA, Fox WW, Abmann SH, Eds. Fetal and neonataly physiology. Philadelphia: Saunders 2004; pp 784-94.

[7] Ballow M, Cates KL, Rowe JC, Goetz C, Desbonnet C. Development of the immune system in very low birth weight (less than $1500 \mathrm{~g}$ ) premature infants: concentration of plasma immunoglobulins and patterns of infections. Pediatr Res 1986; 20: 899-904.
[8] De Sierra TM, Kumar ML, Wasser TE, Murphy BR, Subbarao EK. Respiratory syncytial virus-specific immunoglobulins in preterm infants. J Pediatr 1993; 122: 787-91.

[9] Meert K, Heidemann S, Abella B, Sarnaik A. Does prematurity alter the course of respiratory syncytial virus infection? Crit Care Med 1990; 18: 1357-9.

[10] Resch B, Gusenleitner W, Mueller W. The impact of respiratory syncytial virus infection: a prospective study in hospitalized infants younger than 2 years. Infection 2002; 30: 193-7.

[11] Horn SD, Smout RJ. Effect of prematurity on respiratory syncytial virus hospital resource use and outcomes. J Pediatr 2003; 143: 133-41.

[12] Stevens TP, Sinkin RA, Hall CB, Maniscalco WM, McConnochie KM. Respiratory syncytial virus and premature infants born at 32 weeks' gestation or earlier: hospitalization and economic implications of prophylaxis. Arch Pediatr Adolesc Med 2000; 154: $55-61$.

[13] American Academy of Pediatrics. Committee on Infectious Disease and Committee on Fetus and Newborn. Prevention of Respiratory Syncytical Virus Infections: Indications for the Use of Palivizumab and Update on the Use of RSV-IGIV. Pediatrics 1998; 102: 1211-6.

[14] Carbonell-Estrany X, Quero J, Bustos G, et al. Rehospitalization because of respiratory syncytial virus infection in premature infants younger than 33 weeks of gestation: a prospective study. IRIS Study Group. Pediatr Infect Dis J 2000; 19: 592-7.

[15] Resch B, Pasnocht A, Gusenleitner W, Mueller W. Rehospitalizations for respiratory disease and respiratory syncytial virus infection in preterm infants of 29-36 weeks gestational age. J Infect 2005; 50: 397-403.

[16] Law BJ, Langley JM, Allen U, et al. The Pediatric Investigators Collaborative Network on Infections in Canada study of predictors of hospitalization for respiratory syncytial virus infection for infants born at 33 through 35 completed weeks of gestation. Pediatr Infect Dis J 2004; 23: 806-14.

[17] Figueras-Aloy J, Carbonell-Estrany X, Quero-Jiménez J, et al; IRIS Study Group. FLIP-2 Study: risk factors linked to respiratory syncytial virus infection requiring hospitalization in premature infants born in Spain at a gestational age of 32 to 35 weeks. Pediatr Infect Dis J 2008; 27: 788-93.

[18] Gunville CF, Sontag MK, Stratton KA, et al. Scope and impact of early and late preterm infants admitted to the PICU with respiratory illness. J Pediatr 2010; 157: 209-14. 
[19] Thomas W, Speer CP. Bronchopulmonary dysplasia: epidemiology, pathogenesis and treatment. Monatsschrift Kinderheilkd 2005; 153: 211-9

[20] Groothuis JR, Gutierrez KM, Lauer BA. Respiratory syncytial virus infection in children with bronchopulmonary dysplasia. Pediatrics 1988; 82: 199-203.

[21] The PREVENT Study Group. Reduction of respiratory syncytial virus hospitalization among premature infants and infants with bronchopulmonary dysplasia using respiratory syncytial virus immune globulin prophylaxis. Pediatrics 1997; 93: 93-7.

[22] The IMpact- RSV Study group. Palivizumab, a humanized respiratory syncytial virus monoclonal antibody, reduced hospitalization from respiratory syncytial virus infection in high-risk infants. Pediatrics 1998; 102: 531-7.

[23] Thomas M, Bedford-Russell A, Sharland M. Hospitalization for RSV infection in ex-preterm infants-implications for use of RSV immune globulin. Arch Dis Child 2000; 83: 122-7

[24] Greenough A, Cox S, Alexander J, et al. Health care utilisation of infants with chronic lung disease, related to hospitalisation for RSV infection. Arch Dis Child 2001; 85: 463-8.

[25] Liese JG, Grill E, Fischer B, Roeckl-Wiedmann I, Carr D, Belohradsky BH; Munich RSV Study Group. Incidence and risk factors of respiratory syncytial virus-related hospitalizations in premature infants in Germany. Eur J Pediatr 2003; 162: 230-6.

[26] Simon A, Ammann RA, Wilkesmann A, et al; DSM RSV Paed Study Group. Respiratory syncytial virus infection in 406 hospitalized premature infants: results from a prospective German multicentre database. Eur J Pediatr 2007; 166: 1273-83.

[27] MacDonald NE, Hall CB, Suffin SC, Alexson C, Harris PJ, Manning JA. Respiratory syncytial viral infection in infants with congenital heart disease. N Engl J Med 1982; 307: 397-400.

[28] Navas L, Wang E, de Carvalho V, Robinson J. Improved outcome of respiratory syncytial virus infection in a high-risk hospitalized population of Canadian children. Pediatric Investigators Collaborative Network on Infections in Canada. J Pediatr 1992; 121: 348-4.

[29] Wang EE, Law BJ, Stephens D. Pediatric Investigators Collaborative Network on Infections in Canada (PICNIC) prospective study of risk factors and outcomes in patients hospitalized with respiratory syncytial viral lower respiratory tract infection. J Pediatr 1995; 126: 212-9.

[30] American Academy of Pediatrcs. Committee on Infectious Disease and Committee on Fetus and Newborn. Prevention of respiratory syncytial virus infection: indication for the use of palivizumab and update on the use of RSV. IGIV. J Pediatr 1998; 102: 1211-6.

[31] Feltes TF, Cabalka AK, Meissner HC, et al. Cardiac Synagis Study Group. Palivizumab prophylaxis reduces hospitalization due to respiratory syncytial virus in young children with hemodynamically significant congenital heart disease. J Pediatr 2003; 143: $532-40$.

[32] American Academy of Pediatrics. Committee on Infectious Disease and Committee on Fetus and Newborn. Revised indications for the use of palivizumab and respiratory syncytial virus immunoglobulin intravenous for the prevention of respiratory syncytial virus infections. Pediatrics 2003; 112: 1442-6.

[33] American Academy of Pediatrcs. Committee on Infectious Disease and committee on Fetus and Newborn. Modified recommandations for the use of palivizumab for prevention of respiratory syncytial virus infections. Pediatrics 2009; 124: 1694-701.

[34] Cohen SA, Zanni R, Cohen A, Harrington M, VanVeldhuisen P, Boron ML; Palivizumab Outcomes Registry Group. Palivizumab use in subjects with congenital heart disease: results from the 20002004 Palivizumab Outcomes Registry. Pediatr Cardiol 2008; 29: $382-7$.

[35] Chang RK, Chen AY. Impact of palivizumab on RSV hospitalizations for children with hemodynamically significant congenital heart disease. Pediatr Cardiol 2010; 31: 90-5.

[36] Kristensen K, Stensballe LG, Bjerre J, et al. Risk factors for respiratory syncytial virus hospitalisation in children with heart disease. Arch Dis Child 2009; 94: 785-9.

[37] Resch B, Manzoni P, Lanari M. Severe respiratory syncytial virus (RSV) infection in infants with neuromuscular diseases and immune deficiency syndromes. Paediatr Respir Rev 2009; 10: 148-53.
[38] Boeckh M, Berrey MM, Bowden RA, Crawford SW, Balsley J, Corey L. Phase 1 evaluation of the respiratory syncytial virusspecific monoclonal antibody palivizumab in recipients of hematopoietic stem cell transplants. J Infect Dis 2001; 184: $350-4$.

[39] Thomas NJ, Hollenbeak CS, Ceneviva GD, Geskey JM, Young MJ. Palivizumab prophylaxis to prevent respiratory syncytial virus mortality after pediatric bone marrow transplantation: a decision analysis model. J Pediatr Hematol Oncol 2007; 29: 227-32.

[40] Hall CB, Powell KR, MacDonald NE, et al. Respiratory syncytial viral infection in children with compromised immune function. $\mathrm{N}$ Engl J Med 1986; 315: 77-81.

[41] Cole PD, Suh JS, Onel K, Stiles J, Armstrong D, Dunkel IJ. Benign outcome of RSV infection in children with cancer. Med Pediatr Oncol 2001; 37: 24-9.

[42] King JC Jr, Burke AR, Clemens JD, et al. Respiratory syncytial virus illnesses in human immunodeficiency virus- and non-infected children. Pediatr Infect Dis J 1993; 12: 733-9.

[43] Madhi SA, Schoub B, Simmank K, Blackburn N, Klugman KP. Increased burden of respiratory viral associated severe lower respiratory tract infections in children infected with human immunodeficiency virus type-1. J Pediatr 2000; 137: 78-84.

[44] Bloemers BLP, van Furth AM, Weijerman ME, et al. A novel risk factor for respiratory syncytical virus bronchiolitis- A prospective birth cohort study. Pediatrics 2007; 120: 1076-81.

[45] Bloemers BLP, van Furth AM, Weijerman ME, et al. High incidence of recurrent wheeze in children with Down Syndrome with and without previous respiratory syncytial virus lower respiration tract infection. Pediar Infect Dis J 2010; 29: 39-42.

[46] Law B, Mac Donald N, Langley J. Severe respiratory syncytial virus infection among otherwise healthy prematurely born infants: what are we trying to prevent? Paediatr Child Health 1998; 3: 402-4.

[47] Simoes EA. Environmental and demographic risk factors for respiratory syncytial virus lower tract disease. J Pediatr 2003; 143: $118-26$.

[48] Figueras-Aloy J, Carbonell-Estrany X, Quero J; IRIS Study Group. Case-control study of the risk factors linked to respiratory syncytial virus infection requiring hospitalization in premature infants born at a gestational age of 33-35 weeks in Spain. Pediatr Infect Dis J 2004; 23: 815-20.

[49] Simoes EA, King SJ, Lehr MV, Groothuis JR. Preterm twins and triplets: a high- risk group for severe respiratory syncytial virus infection. Am J Dis Child 1993; 147: 303-6.

[50] Glezen WP, Paredes A, Allison JE, Taber LH, Frank AL. Risk factors for respiratory syncytial virus infection for infants from low- income families in relation to age, sex, ethnic group, and maternal antibody level. J Pediatr 1981; 98: 708-15.

[51] Jansson L, Nilson P, Olsson M. Socioeconomic enviromental factors and hospitalization for acute bronchiolitis during infancy. Acta Paediatr 2002; 91: 335-8.

[52] Anderson LJ, Parker RA, Strikas RA, Farrar JA, Gangarosa EJ, Keyserling HL. Day- care centre attendance and hospitalization for lower respiratory tract illness. Pediatrics 1988; 83: 300-8.

[53] Mc Connochie KM, Roghmann KJ. Parental smoking, presence of older siblings, and family history of asthma increase risk of bronchiolitis. Am J Dis Child 1986; 140: 806-12.

[54] Holberg CJ, Wright AL, Martinez FD, Ray CG, Taussig LM, Lebowitz MD, Group Health Medical Associates. Risk factors for respiratory syncytial virus- associated lower respiratory illnesses in the first year of life. Am J Epidemiol 1991; 133: 1135-51.

[55] Meissner HC. Commentary: The unresolved issue of risk factors for hospitalization of infants with respiratory syncytial virus infection born after 33-35 weeks gestation. Pediatr Infect Dis 2004; 23: 821-3.

[56] Stensballe LG, Ravn H, Kristensen K, Meakins T, Aaby P, Simoes EA. Seasonal variation of maternally derived respiratory syncytial virus antibodies and association with infant hospitalizations for respiratory syncytial virus. J Pediatr 2009; 154: 296-8.

[57] Stensballe LG, Devasundaram JK, Simoes EA. Respiratory syncytial virus epidemics: the ups and downs of a seasonal virus. Pediatr Infect Dis J 2003; 22: 21-32.

[58] Choudhuri JA, Ogden LG, Ruttenber AJ, Thomas DS, Todd JK, Simoes EA. Effect of altitude on hospitalizations for respiratory syncytial virus infection. Pediatrics 2006; 117: 349-56. 
[59] Simões EA, Carbonell-Estrany X, Fullarton JR, et al. European RSV Risk Factor Study Group. A predictive model for respiratory syncytial virus (RSV) hospitalisation of premature infants born at 33-35 weeks of gestational age, based on data from the Spanish FLIP Study. Respir Res 2008; 9: 78.

[60] Stensballe LG, Fullarton JR, Carbonell-Estrany X, Simões EA. Population based external validation of a European predictive model for respiratory syncytial virus hospitalization of premature infants born 33 to 35 weeks of gestational age. Pediatr Infect Dis J 2010; 29: 374-6.

[61] Carbonell-Estrany X, Simões EA, Fullarton JR, Ferdynus C, Gouyon JB; European RSV Risk Factor Study Group. Validation of a model to predict hospitalization due to RSV of infants born at 33-35 weeks' gestation. J Perinat Med 2010; 38: 411-7.

[62] Simões EA, Carbonell-Estrany X, Fullarton JR, Rossi GA, Barberi I, Lanari M, European RSV Risk Factor Study Group. European risk factors' model to predict hospitalization of premature infants born 33-35 weeks' gestational age with respiratory syncytial virus: validation with Italian data. J Matern Fetal Neonatal Med 2011; 24: 152-7.

[63] Simões EA, Carbonell-Estrany X, Fullarton JR, Rossi GA, Barberi I, Lanari M; European RSV Risk Factor Study Group. Development and validation of a risk scoring tool to predict respiratory syncytial virus hospitalization in premature infants born at 33 through 35 completed weeks of gestation. Med Decis Mak 2008; 28: 471-80.

[64] Paes B, Steele S, Janes M, Pinelli J. Risk-Scoring Tool for respiratory syncytial virus prophylaxis in premature infants born at 33-35 completed weeks' gestational age in Canada. Curr Med Res Opin 2009; 25: 1585-91.

[65] Paes B, Cole M, Latchman A, Pinelli J. Predictive value of the respiratory syncytial virus risk-scoring tool in the term infant in Canada. Curr Med Res Opin 2009; 25: 2191-6.

(C) Sommer et al.; Licensee Bentham Open.

This is an open access article licensed under the terms of the Creative Commons Attribution Non-Commercial License (http://creativecommons.org/licenses/ by-nc/3.0/) which permits unrestricted, non-commercial use, distribution and reproduction in any medium, provided the work is properly cited. 